July 1963112

\title{
Pressure Drop and Distribution of Moisture Content in Layers of Rayon Staple Dried by Throughflow of Air
}

\author{
By Hisao Kono, Member, TMSJ
}

Toho Rayon Co., Ltd., Tokushima Plant, Tokushima.

\begin{abstract}
The drying the falling-rate period of drying is known to be approximately proportional to the moisture content $W$.

$$
\frac{\partial W}{\partial \theta}=-K W
$$

where $W:$ moisture content

$\theta$ : time

(kg water/kg dried fiber)

$K$ : proportionality constant

$(\mathrm{min})$

$(1 / \mathrm{min})$

The author has found that $K$ is a function of the temperature and humidity of drying air; and that $\frac{\partial K}{\partial w}$ decreases at a rate proportional to $R / G$, where $R$ is the drying rate, $\partial W / \partial \theta$ and $G$ is the mass velocity of drying air $\left(\mathrm{kg} / \mathrm{m}^{2}\right)$. This relation is expressible thus by equation :

$$
\frac{\partial K}{\partial w}=\frac{J}{G}-\frac{\partial W}{\partial \theta}
$$

where $J$ is a proportionality constant.

The two partial differential equations were integrated numerically with results which a greed well with experimental data.

The pressure drop across fiber layers cannot be represented by a single coefficient of resistance, becuause fiber layers are compressed in varying degrees by the applied air pressure.

The mean solid fraction $(1-\bar{\varepsilon})$ in fiber layers compressed by air pressure was obtained from the compressional characteristics of fiber layers compressed by uniform pressure so as to modify the above-mentioned coefficient of resistance for the pressure drop. The coefficient of friction when divided by a certain power of $(1-\bar{\varepsilon})$ is a relatively constant value.
\end{abstract}

\section{Introduction}

Rayon staple fibers are usually dried with throghflow dryers having band conveyers. Dried fiber layers, however, contain unevenness of water content induced by variations in thickness, in bulk density and in air velocity. The water content also varies according to the distance from upper surface.

Unevenness of water content due to many of the above-mentioned causes cannot be treated theoretically, but it is within the realm of possibility to analyse mathematically the effect of depth on local water content.

There have been published researches on throughflow drying. None of the few published works, except Van Arsdel's[1] treat the effect of depth on water content. He analyzed the unsteady transfer of heat and mass during through-flow drying. $\mathrm{He}$ derived a set of simultaneous partial differential equations consisting of four equations, and integrated them numerically by using, as an example, the drying of a potato cut in half.

The mechanism of heat transfer in a large object like a potato is comparatively simple. Fiber layers, however, are not subject to analysis by methods like Van Arsdel's because not even the temperature of individual fibers can be measured.

It is common knowledge that the drying rate of rayon staple fiber layers in the falling-rate period is approximately proportional to their water content.[2] 
The author, assuming that its proportionality constant was a function of layer thickness alone, derived a set of simultaneous partial differential equations consisting of two equations and integrated them numerically with results which agreed well with experimental data.

We report also on the pressure drop across fiber layers with consideration given to its compression by air pressure.

\section{Experiment Apparatus Used and Conditions of Experiment}

A sketch of the experimental apparatus is given in Fig. 1. The duct, made of a tin plate, is $100 \mathrm{~mm}$ in diameter. The drying chamber, built of transparent vinyl chloride boards, is $200 \mathrm{~mm}$ in diameter. The heater is a fin tube, with a heat-transferring area of $3 \mathrm{~m}^{2}$. The pressure and quantity of air in the blower are $500 \mathrm{~kg} / \mathrm{m}^{2}$ and $30 \mathrm{~m}^{3} / \mathrm{min}$. A damper controls air velocity and adjusts the intake and exhaust of air for humidity control.

The conditions of the experiment were as follows: We initially made several groups of rayon fibers for testing, each group about $100 \sim 500 \mathrm{~g}$ in weight, inserted red strings between them as identifying marks and dried the fibers in the drying chamber. During drying, samples were extracted from the marked positions and their water content was measured. The identifying strings were inserted into fiber layers, because otherwise drying of fibers and the compressive force of the air current made of the fiber layers shrink, thus making the sampling position difficult to identify.
The weight and the quality of the bulks, the temperature, humidity and the mass velocity of air were varied during the experiment. The conditions of the experiment are summed up in Table 1 together with the experimental results.

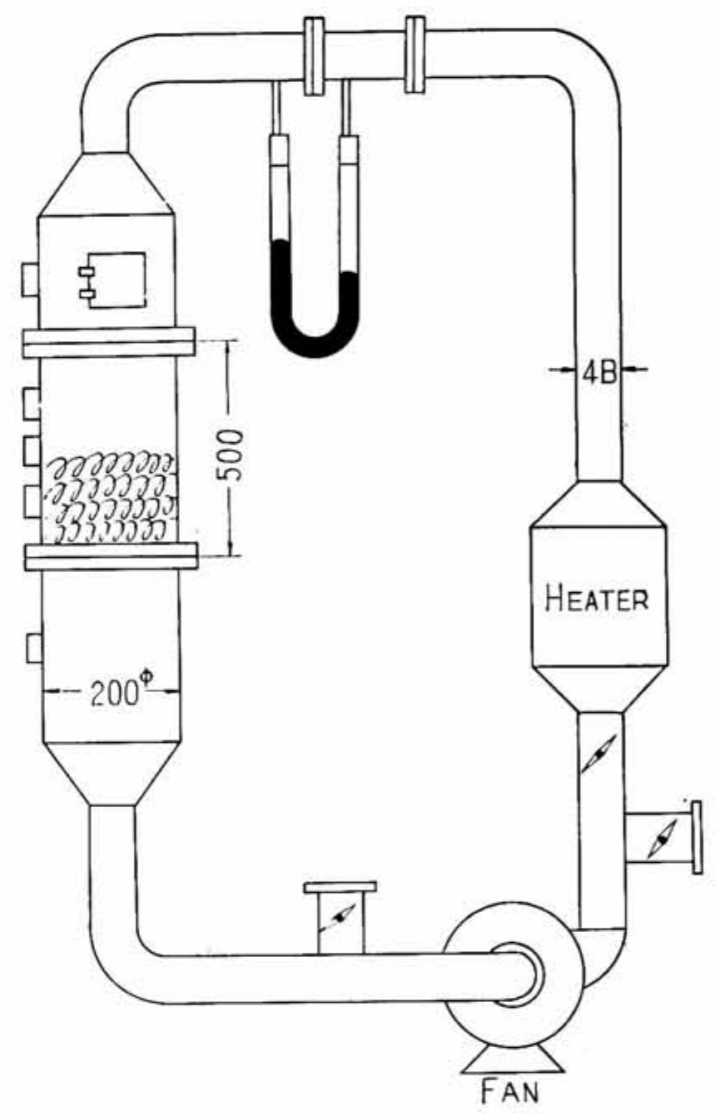

Fig. 1 Experimental Apparatus

Table 1 Conditions of drying and values of $K_{0}$, and $J$

\begin{tabular}{|c|c|c|c|c|c|c|c|c|c|c|}
\hline \multirow{2}{*}{\multicolumn{2}{|c|}{ Nomenclatures }} & \multirow{2}{*}{ Sign } & \multirow{2}{*}{ Unit } & \multicolumn{7}{|c|}{ No. of experiments } \\
\hline & & & & 1 & 2 & 3 & 4 & 5 & 6 & 7 \\
\hline \multicolumn{2}{|c|}{ Number of fiber layers } & & $(-)$ & 2 & 2 & 3 & 1 & 1 & 1 & 1 \\
\hline \multicolumn{2}{|c|}{ Thickness of a layer } & $w$ & $\left(\mathrm{~kg} / \mathrm{m}^{2}\right)$ & 7.65 & 6.60 & 6.60 & 2.31 & 2.27 & 1.95 & 2.25 \\
\hline \multirow{6}{*}{ 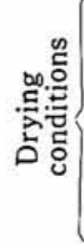 } & Dry bulb temp. & $t$ & $\left({ }^{\circ} \mathrm{C}\right)$ & 84 & 80 & 82 & 70 & 66 & 74 & 79 \\
\hline & Wet bulb temp. & $t_{s}$ & $\left({ }^{\circ} \mathrm{C}\right)$ & 36 & 36 & 37.5 & 32 & 30 & 38 & 34 \\
\hline & Absolute humidity & $H$ & (kg water $/ \mathrm{kg}^{*}$ ) & 0.019 & 0.021 & 0.023 & 0.015 & 0.012 & 0.028 & 0.015 \\
\hline & $\begin{array}{l}\text { Saturated absolute } \\
\text { humidity at } t_{s}\end{array}$ & $H_{8}$ & (kg water $/ \mathrm{kg}^{*}$ ) & 0.0395 & 0.0395 & 0.042 & 0.031 & 0.027 & 0.044 & 0.034 \\
\hline & Density of air & $r$ & $\left(\mathrm{~kg} / \mathrm{m}^{3}\right)$ & 1.02 & 0.97 & 1.02 & 1.00 & 0.96 & 0.98 & 0.96 \\
\hline & Mass velocity of air & $G$ & $\left(\mathrm{~kg} / \mathrm{m}^{2} \mathrm{~min}\right)$ & 35.3 & 27.6 & 24.8 & 39.7 & 29.4 & 57.2 & 60.0 \\
\hline \multirow{3}{*}{ 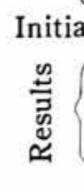 } & 1 water content & $W_{0}$ & $(-)$ & 1.03 & 1.40 & 1.40 & 1.07 & 1.09 & 1.23 & 1.14 \\
\hline & $\begin{array}{l}\text { Drying rete pro- } \\
\text { portionality constant }\end{array}$ & $K_{0}$ & $(1 / \mathrm{min})$ & 0.250 & 0.200 & 0.202 & 0.310 & 0.215 & 0.419 & 0.380 \\
\hline & $\begin{array}{l}\text { Interval coefficient } \\
\text { for fiber layers }\end{array}$ & $J$ & $(\min )$ & 58.0 & 75.3 & 79.0 & 68.5 & 45.0 & 146.2 & 53.7 \\
\hline
\end{tabular}


Fiber layers used for the drying experiment were all $2 \mathrm{~d} \times 57$ in dimensions and had been selected from uniform and lumpless fiber layers in the usual process. The pressure drop across fiber layers was obtaind by measuring the static pressures above and below the fiber layers and taking the difference between them. In addition to the fiber layers taken from the production process, three other fiber samples were similarly tested to obtain a better understanding of the nature of the pressure drop. The four samples tested for pressure drop are:

A) Fiber layers fully opened manually, $2 \mathrm{~d} \times$ $61 \mathrm{~mm}$, their density being $9.0 \mathrm{~kg} / \mathrm{m}^{3}$.

B) Fiber layers used in the drying experiment.

C) Fiber layers opened with an S.R.R.L opener, $3 \mathrm{~d} \times 2-1 / 4^{\prime \prime}$, their bulk density $12.2 \mathrm{~kg} / \mathrm{m}^{3}$.

D) Fiber layers opened with a hopper feeder and hopper bale breaker, $3 \mathrm{~d} \times 2-1 / 4^{\prime \prime}$, their bulk density being $24.0 \mathrm{~kg} / \mathrm{m}^{8}$.

The fiber layers used in the pressure drop experiment had been compressed to half and doubled in their bulk density. The bulk compressional characteristics of these four kinds of fiber layers were measured by Takigawa's method[3] so as to modify the drag coefficient which indicated their pressure loss.

\section{Experimental Results}

Table 1 shows the results of the through-flow drying experiment. Figs. 2 and 3 show changes in water content during drying and compare them with a theoretical equation which will be dealt with later.

As is clear from these figures, the decrease in water content occurs in the top layer first and subsequently propagates to the lower layers in the same manner as in the absorption on a fix bed. This drying mechanism is confirmed also on the temperature curve of dried fiber layers in Fig. 2.

It is particularly interesting that the temperature of the bottom layers is always constant and equals the wet bulb temperature of drying air before it enteres the fiber layers.

Drying air enters fiber layers through the top layer and decreases in temperature and increases in humidity as it dries the top layer and descends to lower.

However, the wet bulb temperature of drying air hardly changes, because the change induced by

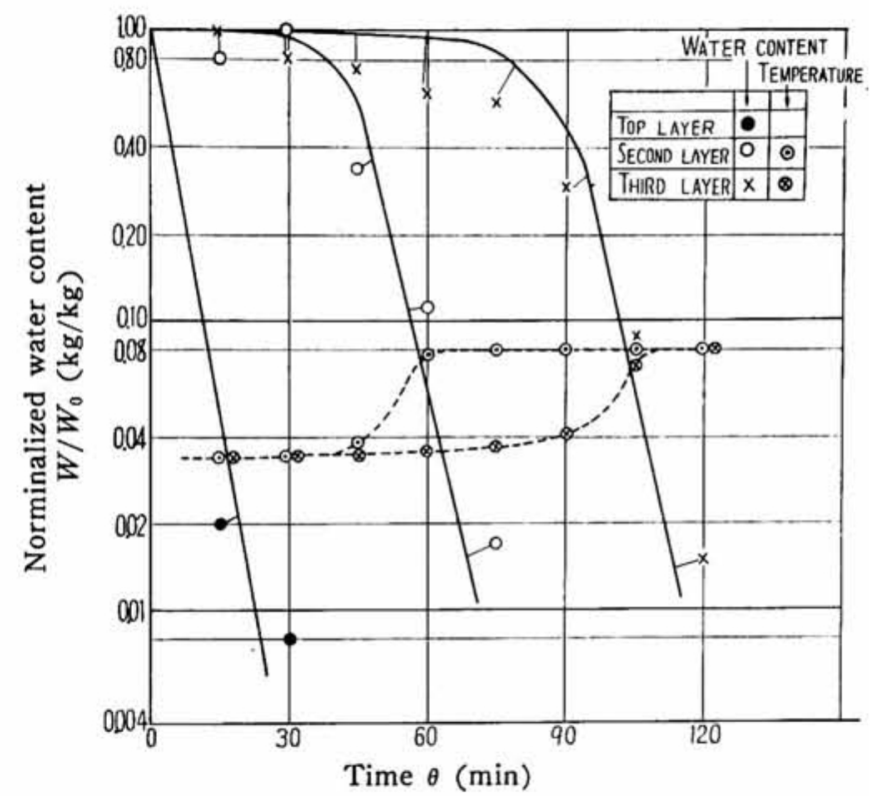

Test No. 2

Fiber $2 \mathrm{~d} \times 57 \mathrm{~mm}$

Conditions of air as it enters fiber layers

Temperature $80^{\circ} \mathrm{C}$

Absolute humidity
\[ =75.3(\mathrm{~min}) \]

Layer thickness

$6.6 \mathrm{~kg} / \mathrm{m}^{2}(208 \mathrm{~g}) \times 2 \quad$ Flow rate $27.6 \mathrm{~kg} / \mathrm{m}^{2} \mathrm{~min}$

Initial water content $140 \%$

Fig. 2 Changes in water content and temperature with time (I)

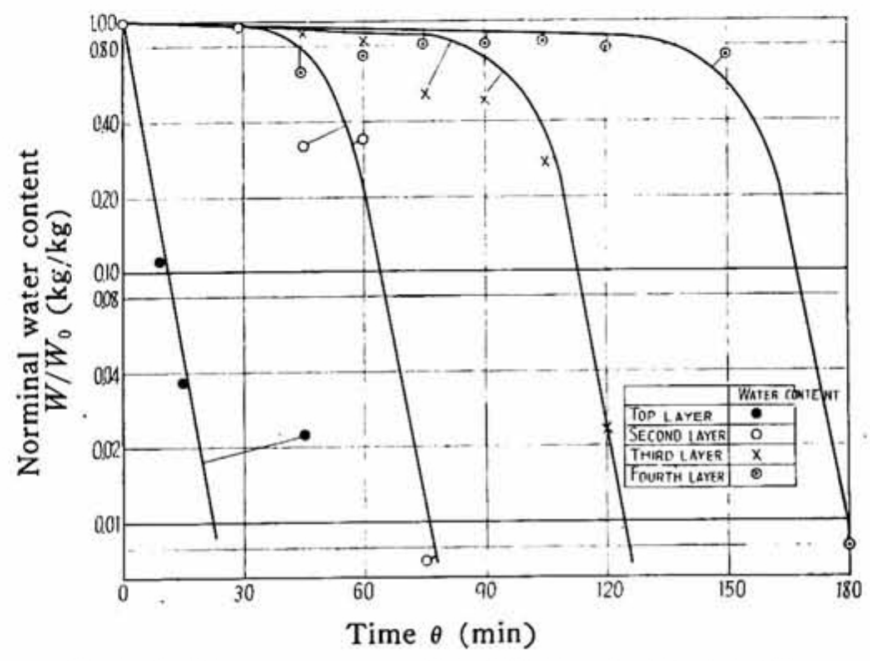

Test No. 2

Conditions of air it enters fiber layers

Fiber $2 \mathrm{~d} \times 57 \mathrm{~mm}$

Temperature $82^{\circ} \mathrm{C}$ $k_{0}=0.202(1 / \mathrm{min})$

Absolute humidity $0.023 \mathrm{~kg} / \mathrm{kg}^{*}$ $A=79.0$ (min)

Layer water

$6.6 \mathrm{~kg} / \mathrm{m}^{2}(208 \mathrm{~g}) \times 3 \quad$ Flow rate $24.8 \mathrm{~kg} / \mathrm{m}^{2} \mathrm{~min}$ Initial water content $140 \%$

Fig. 3 Changes in water content with time 
drying is almost adiabatic along the isoenthalpic curve. This explains why the temperature of the bottom layer is almost constant.

Fig. 4 shows the pressure drop in air blowing through fiber layers. In a range of $0.5 \sim 2.0 \mathrm{~m} / \mathrm{sec}$ of air velocity, the pressure drop versus the air velocity curve of both logarythm scales is almost straight and parallel, the exponent of air velocity, $v$, being about 1.5. With air velocity less than $0.5 \mathrm{~m} / \mathrm{sec}$, the exponent of $v$ decreases to about 0.3 . In a range of $0.5 \sim 2.0 \mathrm{~m} / \mathrm{sec}$ air velocity, the pressure drop is also a function of the thickness of fiber mats and is expressible by equation:

$$
P=10 w^{1.42} v^{1.48}
$$

where $P$ : pressure drop across fiber layers $\left(\mathrm{kg} / \mathrm{m}^{2}\right)$

$w$ : thickness, in weight, of fiber mat $\left(\mathrm{kg} / \mathrm{m}^{2}\right)$ $v$ : air velocity $(\mathrm{m} / \mathrm{sec})$

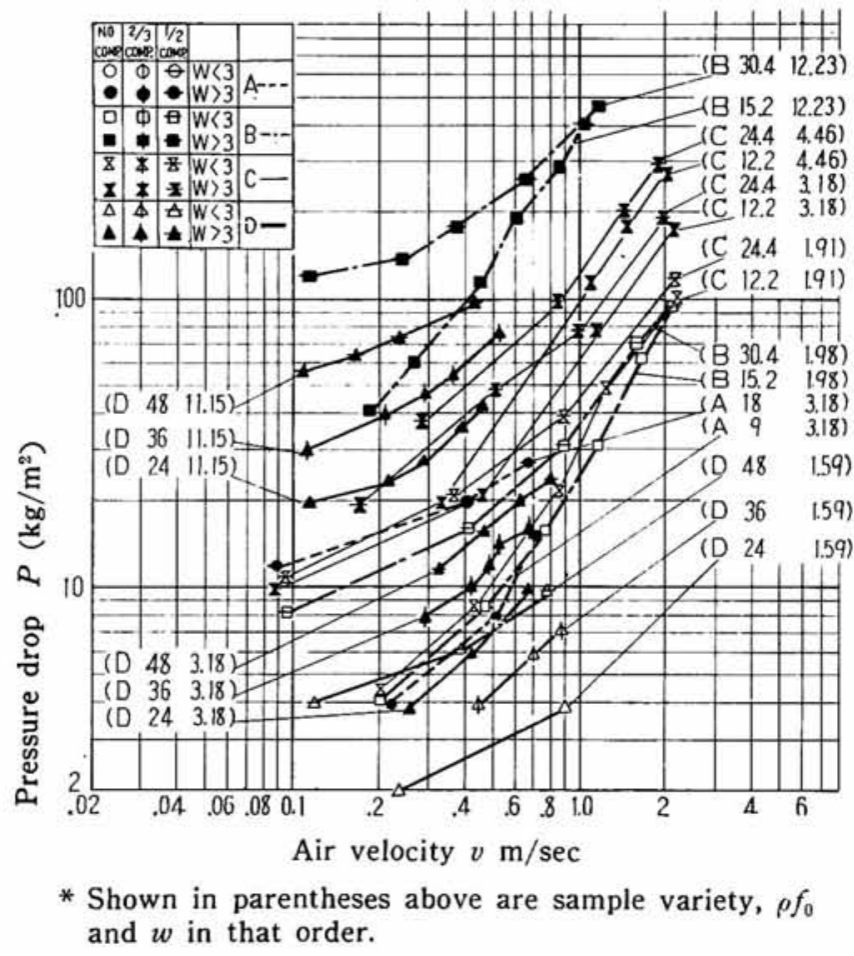

Fig. 4 Pressure drop across fiber layers and air veiocity

\section{Discussion and Theoretical Equation of Drying}

\section{4-1. Drying rate}

The water content of rayon staple fiber layers before industrial dgying is about $120 \%$ (dry base). Hence its drying begins at a falling-rate almost from the beginning. The drying rate of a fine material like fibers in the falling-rate period can be approximately given by the equation:

$$
\frac{\partial W}{\partial \theta}=-K\left(W-W_{c}\right)
$$

where $W$ : drying water content

$\theta$ : time

$K$ : proportionality constant of drying rate

$(1 / \mathrm{min})$

At the critical water content $W_{e}$, the fallingrate drying and the constant-rate drying share an identical drying rate. Therefore,

$$
K=\frac{k g_{a}\left(H_{s}-H\right)}{\rho_{f}\left(W_{c}-W_{e}\right)}=\frac{h_{a}\left(t-t_{s}\right)}{\rho_{f} r\left(W_{c}-W_{e}\right)}
$$

where $W_{c}$ : critical water content (-)

$W_{c}$ : equilibrium water content $(-)$

$\rho_{f}$ : bulk density of fiber mat after $\left(\mathrm{kg} / \mathrm{m}^{3}\right)$

$r$ : latent heat of water at drying temper ature $(\mathrm{kcal} / \mathrm{kg})$

$k g_{a}:$ mass transfer capacity coefficient $\left(\mathrm{kg} / \mathrm{hr} \mathrm{m}^{3} \Delta H\right)$

$H s$ : saturated absolute humidity corresponding to temperature of dried material in a constant-rate period

( $\mathrm{kg}$ water $/ \mathrm{kg}$ dry air)

$H$ : absolute humidity of hot air (kg water/kg dry air)

$h_{a}$ : capacity coefficient of heat transfer

$$
\left(\mathrm{kcal} / \mathrm{hr} \mathrm{m}^{3 \circ} \mathrm{C}\right. \text { ) }
$$

$t$ : hot air temperature

$t s$ : temperature of material dried in a constant-rate period $\left({ }^{\circ} \mathrm{C}\right)$

( $\doteqdot$ wet bulb temperature)

Eq.(2) expresses the value of $K$ in a falling-rate period, but the values of $H s$ and $t s$ in the equation do not correspond to the fiber layer's temperature in a falling-rate period, but do correspond to it in a constant-rate period. The temperature of a fiber layer in a constant-rate period approximately equals the wet bulb temperature of drying air.

The drying-induced increase in humidity being an adiabatic change, the wet bulb temperature, even in a fiber layer in a falling-rate period, is approximately equal to the wet bulb temperature of drying air before it enters the fiber layers and is constant. Hence the values of $H s$ and $t s$ in eq.(2) are approximately constant throughout the fiber layers and throughout the drying period.

It follows, then, that $K$ expressed by eq.(2) is the function of only temperature $t$ and humidity $H$ of drying air.

4-2. Decrease in $K$ by passing of air 
By considering a water balance of a small element in a fiber layers in a falling-rate period, we obtain the following equation:

$$
-d\left(H_{s}-H\right) G=(-\partial W / \partial \theta) d w
$$

From eqs.(2) and (3) emerges this equation:

$$
-\frac{\partial K}{\partial w}=\frac{K g a}{\rho_{f}\left(W_{c}-W_{\theta}\right) G}\left(-\frac{\partial W}{\partial \theta}\right)
$$

where $G$ : mass velocity of drying air $\left(\mathrm{kg} / \mathrm{m}^{2} \mathrm{~min}\right)$

By considering a similar heat balance, we derive:

$-C_{p} \cdot G \cdot d\left(t-t_{s}\right)=r(-\partial W / \partial \theta) d w+C\left(\partial t_{m} / \partial \theta\right) d w$

From eqs. (2) and (5) emerges equation (6):

$$
\begin{gathered}
-\frac{\partial K}{\partial w}=\frac{h_{a}}{C_{p \rho_{f}}\left(W_{c}-W_{e}\right) \cdot G}\left\{\left(-\frac{\partial W}{\partial \theta}\right)\right. \\
\left.+\frac{C_{f}}{r}\left(\begin{array}{c}
\partial t_{m} \\
\partial \theta
\end{array}\right)\right\}
\end{gathered}
$$

where $C_{p}$ : specific heat of air

$C_{r}$ : specific heat of wet fiber

$t_{m}$ : temperature of fiber mat in a falling. rate period.

The second term of eq.(6) is much smaller than the first term; the error is below a few per cent, even if the second term is Ignored. Partial-differential equation (7) derived from eqs.(4) and (6) expresses the decrease in $K$ as through-flow drying proceeds:

$$
\frac{\partial K}{\partial w}=\frac{J}{G} \frac{\partial W}{\partial \theta}
$$

where $J$ is a proportional constant.

The value of $J$ is obtainable from either eq. (4) or (6), but the deviation from the value obtained by a theoretical equation will probably be compounded

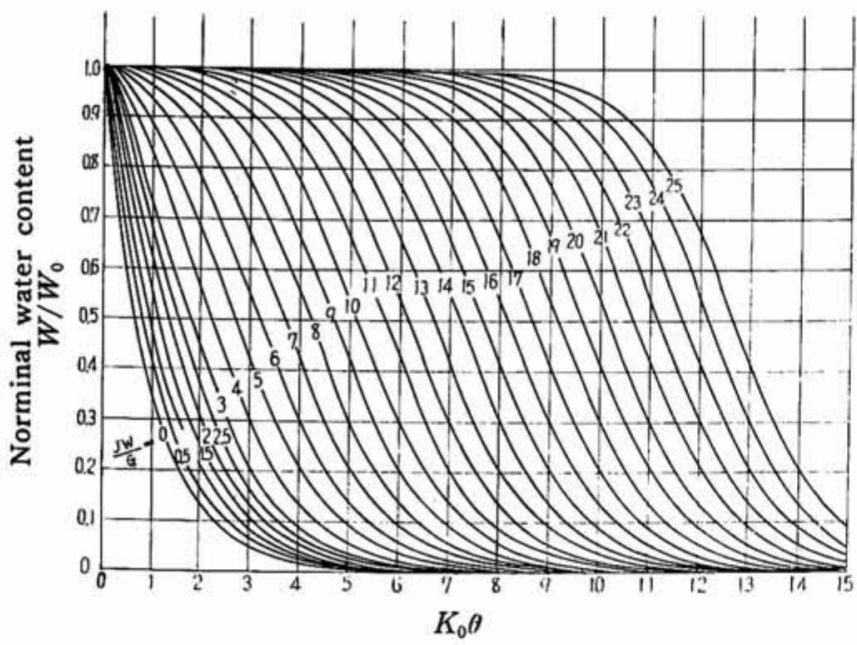

Fig. 5 Changes in local water content with time, theortical values by the error of approximation and unevenness of a fiber mat. Accordingly, we treat $J$ as an experimental constant.

We should be able to obtain an expression of the water content of the fiber layer in any position during by integrating a similtaneous partial differential equations (1) to (7). However, since they defy analytical integration, we integrated them numerically by the following method:

1) Divide time and the thickness of the fiber layers into small elements.

2) Assume that the values of $K$ and $W$ are uniform within a small element.

3) Obtain the values of $K$ and $W$ by integrating the small elements in chronogical order from the upper to lower layers.

The results obtained are given in Fig. 5.

\section{4-3. Discussion}

A comparison of the experimental results and Fig. 5 is made in Figs. 2 and 3 . Assuming that the value of $K$ before drying air enters a fiber mat is $K_{0}$, then the values of $K_{0}$ and $J$ should be obtainable from the condition of drying air and expressible as the function of the fiber denier and bulk density of the fiber layers and as a function of temerature, humidity and quantity of drying air. However, the experiment under review was too limited in range for these relations to be clarified.

The values of $K_{0}$ and $J$ in our experiment are shown in Table 1 , which shows that, in the range our experiment, $K_{0}$ depends almost entirely on the weight velocity of drying air, $G\left(\mathrm{~kg} / \mathrm{m}^{2} \mathrm{~min}\right)$, and is expressible thus:

$$
K_{0}=0.0149 G^{0.8} \quad(1 / \mathrm{min})
$$

The variable most closely correlated to $J$ is $H$. The correlation between $J$ and $G$ is also considerable. A wider range of experiment would be needed to establish these correlations definitely.

\section{Pressure Drop in Air Blowing through Fiber Layers}

\section{5-1. Coefficient of Resistance}

The equation to express pressure drop $P\left(\mathrm{~kg} / \mathrm{m}^{2}\right)$ in a fiber mat, using coefficient $C$ of resistance, is $[4]$ :

$$
P=C \frac{r v_{a}^{2}}{2 g} \frac{A_{p} N}{A}
$$


where $P$ : pressure drop in air blowing through the fiber mat

$\left(\mathrm{kg} / \mathrm{m}^{2}\right)$

$C$ : coefficient of friction for the fiber layers

$r$ : density of air

$v_{a}:$ actual velocity $(=v / \varepsilon)$

$A_{p}$ : area of one fiber projected to the dire ction of flow

$N$ : number of fibers in the fiber layer $(-)$

$A$ : cross sectional area of the fiber mat $\left(\mathrm{m}^{2}\right)$

$\varepsilon$ : porosity of the fiber mat

$g$ : gravitational acceleration

$\left(\mathrm{m} / \mathrm{sec}^{2}\right)$

The following relation about fiber mats is common knowledge :

$$
\begin{aligned}
& l=9 \times 10^{6} \quad w_{A} / D_{c} \\
& D_{e}=9 \times 10^{6} \rho_{s} D_{f}^{2} \pi / 4 \\
& A_{p} N=D_{f} l=9 \times 10^{6} \quad w D_{f} / D c=4 w / \rho_{s} D_{f} \pi
\end{aligned}
$$

where $l$ : total length of all fibers in a fiber mat (m)

$w$ : thickness, in weight, of a fiber mat

$D_{e}$ : denier of a fiber

$D_{j}$ : diameter of a fiber

$\rho_{s}$ : density of a fiber

$\left(\mathrm{kg} / \mathrm{m}^{2}\right)$

In the light of eqs. (9) and (10), the pressure drop in a mat is as follows:

$$
\begin{gathered}
\frac{P}{r}=C \frac{3 \times 10^{3}}{g \sqrt{\pi \rho_{s}}} \frac{w}{\sqrt{D_{e}}} \frac{v^{2}}{\varepsilon^{2}} \\
C=\frac{1}{3} \times 10^{-3} \frac{g \sqrt{\pi \rho_{s}}}{r} \frac{\sqrt{D_{e}}}{w} \frac{\varepsilon^{2} P}{v^{2}}
\end{gathered}
$$

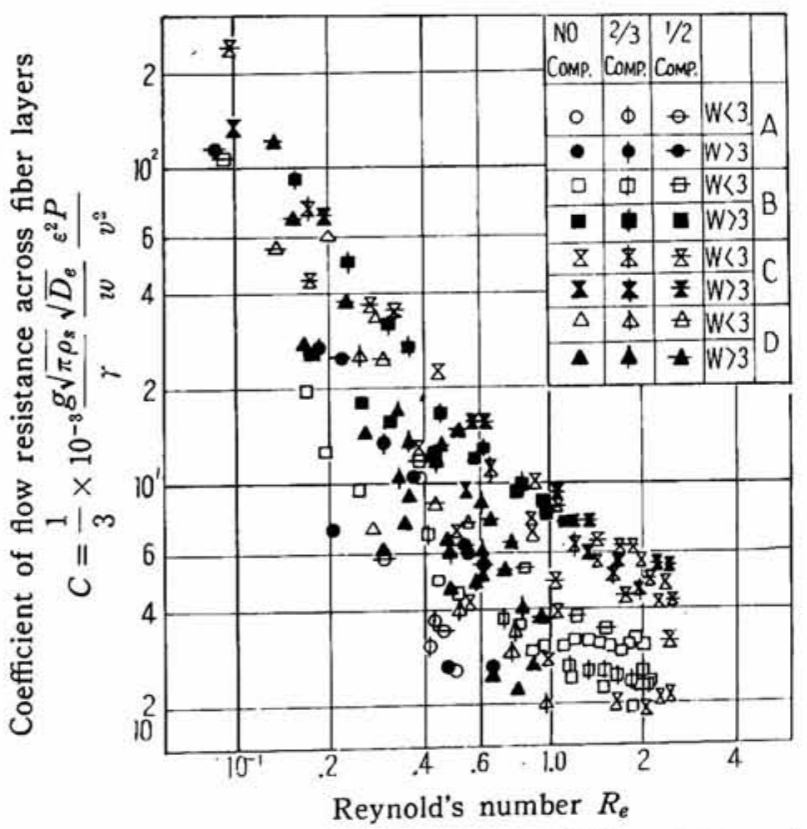

Fig. 6 Coefficient of flow resistance and Reynold's number
The relation between the coefficient of resistance and Reynold's number calculated by eq.(12) is given in Fig. 6, which shows the value of the coefficient is scattered widely.

The coefficient $C$ of friction of fiber layers which, after adequate compression, has a high density and is not affiected by air pressure, is concentrated in the upper part of the group and tends to be almost inversely proportional to Reynold's number, as is stated in Kimura's research[4].

We find from reference[5] that the coefficient of friction for a fiber layer is proportional to a certain power of its solid fraction $(1-\varepsilon)$. The mean solid fraction of a fiber layer compressed by air pressure was measured so as to modify the drag coefficient.

5-2. Mean solid fraction of fiber layers compressed by air blowing through them

5-2-1. Bulk compressional characteristics of rayon staple fiber layers

To know the condition of the compression of the compression of fiber mats by air pressure, we studied the bulk compressional characteristics of of fiber layers compressed by uniform pressure. The bulk compressional characteristics of fiber layers are greatly affected by denier, bulk density and their distribution. A series of experiments revealed that:

a) Young's apparent modulus of a well-opened fiber layer depends only on bulk density, and is expressible by the equation:

$$
E=E_{0} \rho_{r}^{a}
$$

$E$ : Young's modulus

$\rho_{f}$ : bulk density of fiber layers $\left(\mathrm{kg} / \mathrm{m}^{3}\right)$

$E_{0}, a$ : constant

The difference in denier between $2 \mathrm{~d}$ and $3 \mathrm{~d}$ is negligible, and the values of these constants of rayon staple fiber layers of $2 \mathrm{~d}$ or $3 \mathrm{~d}$ are, in the range of our experiments :

$$
\begin{aligned}
& E_{0}=0.00235 \\
& a=3.205
\end{aligned}
$$

Compressional characteristics obtained by compressing the same fiber layers repeatedly show that Young's modulus is the same if bulk density is the same. Therefore, pressure versus volume curves are parallel to each other and are practically identical in shape except for the shift in their vertical positions.

b) Fiber layers in the usual process have many lumps and the distribution of their bulk density is widely scattered. The compressional characteristics of such fiber layers agree well with those of well-opened fiber layers mentioned before, if we 
deduct a certain weight, $L \mathrm{~kg}$, from the actual weight, $f \mathrm{~kg}$, regard the balance, $F \mathrm{~kg}$, as the apparent weight of the mat, and use the apparent bulk density $F / V$ in place of the actual density $f / v$. This procedure is expressible by the following equation:

$$
\begin{aligned}
& \rho_{f}=f / V \\
& \rho_{F}=F / V=(f-L) / V \\
& \rho_{f}=\rho_{F}+L / V=\rho_{F}+\left(\rho_{F} / \rho_{0}\right)\left(L / V_{0}\right)
\end{aligned}
$$

where $\rho_{f}$ : actual bulk density of fiber layers

$f$ : actual weight of fiber layers

$V$ : volume of fiber layers

$\rho_{F}$ : apparent bulk density of fiber layers

$F$ : apparent weight of fiber layers

$L$ : the weight to be substracted from the actual weight to obtain the apparent weight of fiber layers

$\rho_{F 0}$ : apparent bulk density before compression

$V_{0}$ : volume before compression

This is clear from the fact the compressive characteristics of fiber layers from the production process coincide with the characteristics of wellopened layers separately. By shifting the curves for the compressive characteristics horizontally until the curve overlap, we can obtain the value of $F$ and $L$. The values of $L, \rho_{F 0}$ thus obtained are given in Table 2 .

Table 2 Value of $\rho_{f 0}, \rho_{F 0}$ for the fiber layers used in experiments

\begin{tabular}{lccc}
\hline Kinds of mats & $\rho_{f_{0}}=f / V_{0}$ & $L / V_{0}$ & $\rho_{F_{0}}=(f-L) / V_{0}$ \\
\hline A $\quad$ Well-opened manually & 9.0 & 0 & 9.0 \\
B In the usual process & 15.2 & 2.2 & 13.0 \\
$\begin{array}{c}\text { (little lump mat) } \\
\text { C Opened with S.R.R.L. } \\
\text { opener }\end{array}$ & 12.2 & 1.3 & 13.5 \\
D Op0ned with H.F. \& & 24.0 & 5.0 & 19.0 \\
\hline
\end{tabular}

By using the apparent bulk density, the relation between the compressional pressure, $P$, and compressed volume, $V$, is expressible by the following equation :

$$
P=\frac{E_{0} \rho_{F 0}{ }^{a}}{1-a}\left\{1-\left(\frac{V}{V_{0}}\right)^{1-a}\right\}
$$

5-2-2. The mean solid fraction of fiber layers compressed by air through-current
The apparent bulk density of uniformly compressed fiber layers is deducible as follows from eq. (15):

$$
\rho_{F^{r}}=\rho_{F^{r} 0}\left(1+\left\{(a+1) P /\left(E_{0} \rho_{F^{r}}{ }^{a}\right)\right\}\right)^{1 / a-1} \ldots \ldots
$$

By using the relation expressed in eq.(14), we get the actual bulk density $\rho_{f}$ from eq.(16). Although the bulk density of fiber tufts in the usual process is widely scattered, the apparent bulk density $\rho_{F^{0}}$ of a fiber layer compressed by air throughcurrent is almost constant and so is its presumably because fiber layers in the production process are, in fact, fairly uniform in density when a large quantity is considered and the local variations in $\rho_{f}$ are due to the presence of fiber lumps of higher densities.

$L$ mentioned before, therefore, can be considered to be the average contribution of local fiber lumps to the density of fiber layers. Since air flowing through fiber layers is likely to go around local fiber lumps, the pressure drop is presumably controlled not by the actual density but by the apparent density. Therefore, we shall not touch on the actual density.

When fiber layers are compressed by the pressure of air current, the layers are not compressed uniformly. The pressure drop is increasingly large in lower layers and so is fiber compression. The pressure drop $d P$ of air blowing through fiber mats $d w$ thick is given by the following equation:

$$
d P=C \frac{3 \times 10^{3} \gamma}{g \sqrt{\pi \rho_{s} D_{e}}} \frac{v^{2}}{\varepsilon^{2}} d w
$$

Approximately the coefficient $C$ of resistance can be considered to be inversely proportional to Reynold's number if, to simply calculation, compression is left out of consideration. Therefore, assuming $C=C^{\prime} / R_{e}$, then:

$$
\varepsilon d P=C^{\prime} B v d w
$$

where $B=\frac{3 \times 10^{3} \nu \gamma}{g \sqrt{\pi \rho_{\varepsilon} D_{e}} D_{f}}$

$$
\nu \text { : viscosity of air }
$$

Compression in a small part like $d w$ may be considered uniform. Therefore, with the aid of eq.(16), we get the following equation to express porosity :

$$
\varepsilon=1-\frac{\rho_{F}}{\rho_{s}}=1-\frac{\rho_{F 0}}{\rho_{s}}\left(1+\frac{a-1}{E_{0} \rho_{F 0}{ }^{a}} P\right)^{1 / a-1}
$$

Generally, the pressure drop in eq. (18) differs from the compressional pressure $P$ in eq.(16). These two pressures, however, become identical when dis- 
cussed in relation to eq. (19), because in eq. (19) compressive pressure $P$ equals pressure drop. From eqs. (18) and (19) we get the mean porosity of the fiber mat as follows:

$$
\begin{gathered}
\varepsilon=\frac{\int_{\varepsilon} d P}{P}=1-\frac{E_{0} p_{F 0 a}}{a P} \frac{\rho_{F^{0} 0}}{\rho_{s}}\{1+ \\
\left.\left(1+\frac{P}{E_{0} \rho_{F 0}{ }^{a}(a-1)}\right)^{a / a-1}\right\}
\end{gathered}
$$

If follows from this that the mean solid fraction $(1-\bar{\varepsilon})$, is

$$
(1-\bar{\varepsilon})=\frac{E_{0} \rho_{F 0}{ }^{a}}{a P} \frac{\rho_{F 0}}{\rho_{s}}\left\{1+\left(1+\frac{P}{E_{0} \rho_{F 0}{ }^{a} /(a-1)}\right)^{a / a-1}\right\}
$$

The value of $(1-\bar{\varepsilon})$ calculated from eq.(21), using $E_{0}=0.00235, a=3.205$, is shown in Fig. 7 .

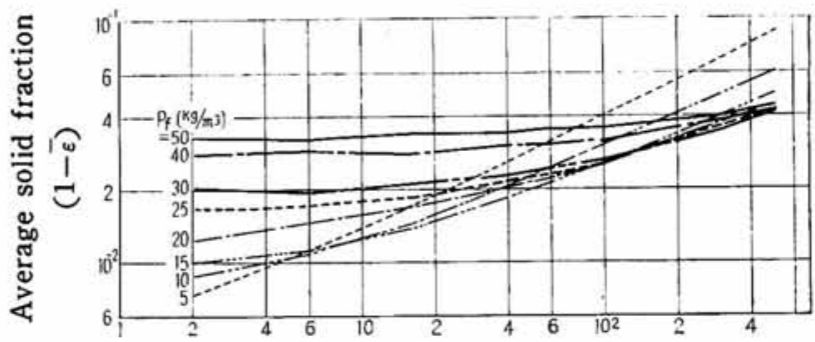

Fig. 7 Effect of pressure drop on mean solid fraction of fiber layers

5-3. Modified coefficient of resistance

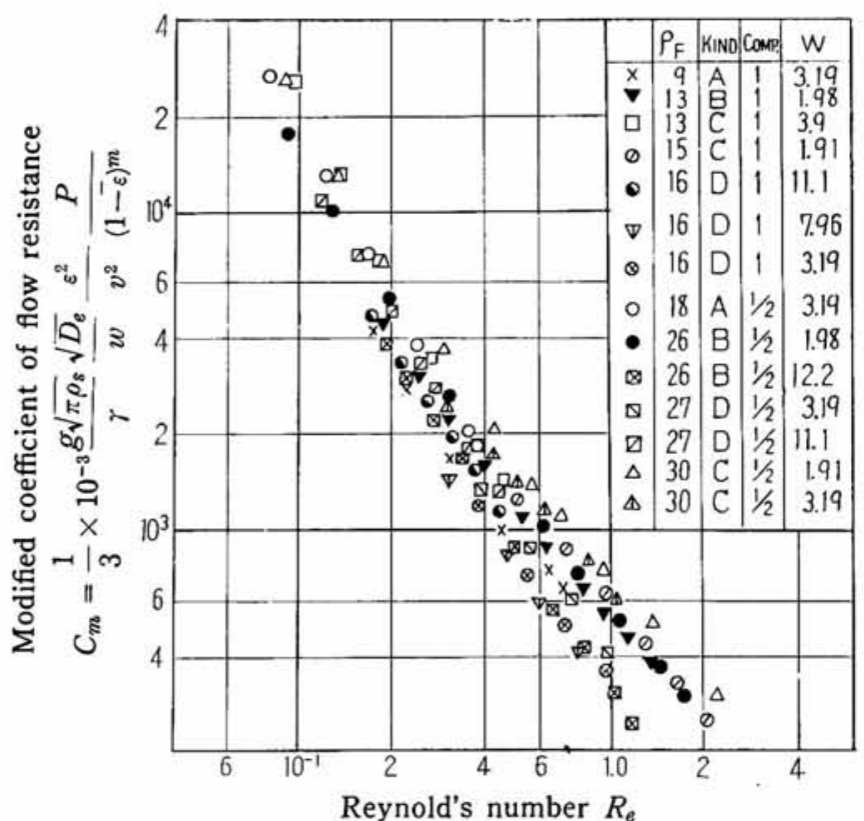

Fig. 8 Modified coefficient of flow resistance and Reynold's number
The modified coefficient of resistance is expres. sible thus:

$$
C_{m}=\frac{1}{3} \times 10^{-3} \frac{g \sqrt{\pi \rho_{s}}}{r} \frac{\sqrt{D_{e}}}{w} \frac{\varepsilon^{2}}{v^{2}} \frac{P}{(1-\bar{\varepsilon})^{m}}
$$

The relation between $C_{m}$ and Reynold's number is given in Fig. 8. The value of $m$ is 1.3 for $2 \mathrm{~d}$ and 1.2 for $3 \mathrm{~d}$, but the the value of $m$ for a number of deniers was unobtainable because our experiments were too limited in the range of deniers.

The equation which fits into the correlation in Fig. 8 is

$$
C_{m}=\frac{320}{R_{0}}+\frac{120}{R_{0}^{2}} \quad 0.1<R_{0}<2.5
$$

$P$ can be computed from eqs. (22) and (23) if $\mathrm{P} /(1-\bar{\varepsilon})^{m}$ is plotted against $\rho_{F 0}$ beforehand. In such computations the initial void fraction $\varepsilon_{0}$ was used in place of $\varepsilon$ in eq.(22), becuase it resulted in negligible errors.

\section{Conclusions}

To obtain the clocal water content in fiber layers dried by air throughflow, a set of simultaneous partial differential equations

$$
\begin{aligned}
& (\partial W / \partial \theta)=-K\left(W-W_{e}\right) \\
& (\partial K / \partial w)=(\partial W / \partial \theta) J / G
\end{aligned}
$$

were integrated numerically with the results shown in Fig. 5. We found by experiment that this agreed well with experimental data.

We also derived an equation to express the pressure drop across fiber layers during drying. In deriving the equation, consideration was given to the compression of the fiber layers by air pressure.

The author is grateful to Prof. T. Otake of Osaka University for his guidance in this study.

\section{Literature cited}

[1] W.B. Van Arsdel; Chem. Eng. Prog. Svmp. Series No. 16, 51, 47 (1955)

[2] A.W. Lykow; "Experimentelle und Theoretische Grun. dlagen der Trocknung"

[3] N. Takigawa and K. Kawamura ; J. Soc. Tex. \& Cell. Ind. (Japan), 17, 271, 301 (1961)

[4] N. Kimura and K. Iinoya; Chem. Eng. (Japan), 23, 792 (1959)

[5] C.Y. Chen; Chem. Rev., 55, 595 (1955) 